\title{
Ensinamentos da prematuridade em tempos de COVID-19
}

\author{
Lessons of prematurity at times of COVID-19
}

\author{
Adriane Gonçalves Salle', Cláudia Simone Silveira dos Santos'², Nina Aguilar Soares \\ e Viviane Salazar
}

\begin{abstract}
Resumo: A pandemia da COVID-19 vem transformando a rotina e a saúde mental da sociedade,e com isso, questões relacionadas ao tema se encontram mais latentes. No hospital, os cuidados intensos aos pacientes e familiares somam-se ao medo, à preocupação, à busca do controle do ambiente diante de uma situação ainda desconhecida, mas de um risco potencial à saúde de todos. As equipes encontram-se mais fragilizadas e necessitando recursos para enfrentar seu estresse e sofrimento. Diante desse cenário, encontram-se nos familiares de bebês prematuros, possíveis aprendizados de resiliência e estratégias de enfrentamento. Este artigo é um relato de experiência de profissionais de Psicologia de uma Unidade de Internação Neonatal de um hospital público e de ensino do Sul do país. Tem como objetivo refletir sobre fenômenos psicológicos vividos na prematuridade e aprendizados possíveis de construir com profissionais de saúde em um cenário de pandemia. Percebe-se que pais de bebês prematuros, por já viverem separações abruptas, incertezas e medos quanto à saúde de seus filhos, possuem estratégias de enfrentamento que conseguem se ampliar para angústias relacionadas à pandemia, adaptando-se mais facilmente ao momento. Pretende-se, com estas observações, voltar a atenção aos pais de prematuros, que muito podem contribuir com intervenções de saúde mental.
\end{abstract}

Abstract: The COVID-19 pandemic has been transforming society's routine and mental health, and with this, issues related to the theme are more latent. At the hospital, intensive care for patients and families is added to fear, concern, the search for control of the environment in the face of an unknown situation, but of a potential risk to the health of all, and the teams are more fragile and needing resources to face their stress and suffering. In view of this scenario, family members of premature babies are able to learn resilience and coping strategies. This article aims to report the experience of Psychology professionals from a Neonatal Inpatient Unit of a public and teaching hospital in the South of the country. Aims to reflect on psychological phenomena experienced in prematurity and possible learnings to build with health professionals in a pandemic scenario. It is noticed that parents of premature babies, for already experiencing abrupt separations, uncertainties and fears about the health of their children, have coping strategies that are able to expand to anguish related to the pandemic, adapting more easily to the moment. It is intended, with these observations, to turn the attention to parents of premature babies, who can contribute a lot with mental health interventions.

Keywords: COVID-19; Prematurity; Health professionals.

Palavras-chave: COVID-19; Prematuridade; Profissionais de saúde.

\footnotetext{
1 Psicóloga do Hospital de Clínicas de Porto Alegre, Especialista em Psicoterapia da Infância e Adolescência (CEAPIA). E-mail: asalle@hcpa.edu.br

2 Psicóloga do Hospital de Clínicas de Porto Alegre, Doutora em Ciências da Saúde: Ginecologia e Obstetrícia (UFRGS). E-mail: csssantos@hcpa.edu.br

3 Psicóloga Residente do campo Atenção Materno-Infantil do Hospital de Clínicas de Porto Alegre. E-mail: nasoares@hcpa.edu.br

4 Psicóloga Residente do campo Atenção Materno-Infantil do Hospital de Clínicas de Porto Alegre. E-mail: visalazar@hcpa.edu.br
} 


\section{Introdução}

Desde o início de 2020, um novo vírus, denominado Coronavírus (Sars-Cov-2), vem causando um número alarmante e súbito de contágio humano e, consequentemente, de óbitos, necessitando que, em março de 2020, a OMS considerasse a COVID-19 (denominação da doença originada pelo contágio do novo Coronavírus) como uma pandemia. (Organização Pan-Americana de Saúde \& Organização Mundial de Saúde, 2020). Até julho de 2020, foram mais de 12 milhões de pessoas contaminadas no mundo, sendo que, destas, mais de 550 mil foram a óbito (John Hopkins University and Medicine, 2020). № Brasil, por sua vez, o número de casos confirmados, de forma acumulada até julho de 2020, ultrapassou 1,7 milhões, havendo mais de 67 mil óbitos causados pela doença (Ministério da Saúde, 2020).

A COVID-19 entrou em nossas vidas sem pedir licença, sem ser anunciada. Chegou e pronto. Imediatamente foram necessárias medidas extremas de cuidados com a saúde de todos. Cuidados estes que vivem num processo de reinvenção contínua, a cada novidade que surge com relação às formas de contágio do novo Coronavírus. Isolamento social, confinamento, distanciamento social: palavras que passaram a fazer parte da vida das pessoas sem muita clareza do significado de cada uma, mas com uma certeza apenas, a de que temos que ficar longe uns dos outros. Famílias, comércio, sociedade tendo que se desacomodar e rapidamente se adaptar ao novo jeito de viver.

Com isso, a saúde mental passou a ser um ponto de importante atenção na sociedade. Percebe-se o quanto lidar com uma doença ainda pouco desvendada pela comunidade científica vem sendo um desafio para todos. Os cuidados constantes, a necessidade do isolamento social, a ruptura de rotinas, prejuízos financeiros, entre outros tantos pontos de impacto por conta desta pandemia vêm influenciando a saúde mental da população, a ponto de se cogitar que os impactos psicológicos na sociedade poderão perdurar para além do período da pandemia, sendo até mesmo mais duradouros e significativos do que as consequências da própria COVID-19 (Schmidt, Crepaldi, Bolze, Neiva-Silva, \& Demenech, 2020).

Em tempos como esse, observamos a continuidade de serviços essenciais, a angústia espalhada e espelhada no rosto dos profissionais e suas famílias, os medos, as incertezas, as dúvidas. Até quando vamos neste compasso? Até quando vamos ter que nos separarmos para, futuramente, ficarmos bem? Quem irá nos dar uma notícia da data que tudo isso irá acabar?

Essas questões nos fizeram associá-las ao nosso trabalho como psicólogas de uma Unidade de Internação Neonatal. Diariamente, intervimos com famílias que, inesperadamente, se deparam com a chegada de um filho prematuro. Junto surgem dúvidas, incertezas, fantasias, imaginário, uma imensa demanda psíquica diante do desconhecido, das necessidades das famílias, dos bebês e da equipe (Rosa \& Gil, 2017). Percebemos, neste ambiente, que diversos fenômenos psicológicos circulam e que poderiam ser foco de atenção e reflexão neste momento. Afinal, o que a prematuridade pode ter a ensinar-nos diante de uma situação tão extrema quanto a de uma pandemia?

\section{Método}

0 proposto artigo se apresenta como um relato de experiência, configurando-se como um estudo descritivo de natureza qualitativa. Para Gil (2002), este modelo de estudo se preocupa em descrever as especificidades de determinado fenômeno, individual ou coletivo. Além de reconhecer as possibilidades entre as dimensões, a pesquisa descritiva também pode fomentar uma nova perspectiva sobre determinado problema.

Trata-se de um relato de experiência das psicólogas da Unidade de Internação Neonatal de um hospital público e de ensino do Sul do país, com base nas vivências em um, no período compreendido entre março e junho de 2020. Os dados foram coletados mediante observação.

\section{Discussão}

\section{A prematuridade enquanto formadora de resiliência}

Desde o início da pandemia, especialmente a partir de março deste ano (2020), observamos 0 quanto os pais dos bebês internados têm vivenciado o momento de maneira mais adaptada do que a equipe de saúde. Há a impressão de que os pais dos bebês ali presentes se comportam como se estivessem desconectados da realidade, e o questionamento sobre como estão se protegendo e protegendo suas famílias se torna cada vez mais presente dentro da unidade. Costumam tomar as medidas de cuidado, mas na maior parte do tempo não demonstram uma fragilidade emocional esperada pela equipe em função da condição de seu bebê somada ao cenário de pandemia. A cada notícia sobre a saúde dos filhos, novas medidas de cuidados. Parecem já fazerem parte e conhecerem melhor essa rotina. Tais observaçōes inquietaram a todos os profissionais de saúde, em especial nós, psicólogas. Passamos a analisar, assim, a relação possível entre a vivência da prematuridade e consequente internação neonatal de um filho na capacidade de resiliência dos pais em cenários desafiadores como de uma pandemia.

A prematuridade entra na vida das famílias, muitas vezes, de forma súbita e não esperada. 0 momento do nascimento se torna algo tenso, preocupante, que desperta medos e expectativas. 0 bebêé separado dos pais abruptamente, fica numa incubadora, the são colocados aportes tecnológicos e o tempo passa num compasso diferente, individual, único. A transferência de confiança faz com que tenham que aprender, internamente, a confiar na equipe e na tecnologia. Sentimentos de tristeza, angústia, culpa e medo surgem neste período, associados ao estigma social relacionado à UTI Neonatal (Silva, Menezes, Cardoso, \& França, 2016). A falta do contato físico, as perguntas sem respostas precisas muitas vezes incrementam a angústia desses pais. Mas escutam da equipe que é assim, aos poucos, com ou sem intercorrências, que a prematuridade se dá, conforme a evolução do bebê. Por vezes escutam limitações terapêuticas, piora significativa, possibilidade de óbito. Recebem as informações e precisam processar, assimilar, compreender, para que possam se sustentar emocional mente ao lado do filho. Ao retornarem para suas casas, compartilham com a família o que escutaram e buscam na mesma 0 apoio necessário, sendo um núcleo de saúde, muitas vezes, para esses pais, atuando como uma referência para compreender a situação de seu filho e entender como atuar durante o processo de internação (Molina, Fonseca, Waidman, \& Marcon, 2009).

Dessa forma, talvez os pais façam uso de estratégias de enfrentamento mais adaptativas a situações de maior demanda emocional e de risco, pois a experiência com a prematuridade faz com que desenvolvam essa capacidade. A vida da família para nessa fase de cuidados, e um novo adaptar-se se instala. Em estudo realizado por Loss, Caprini, Rigoni, \& Andrade (2015) com mães de bebês internados em uma Unidade de Terapia Intensiva Neonatal, foi realizado 
um levantamento acerca das estratégias de enfrentamento utilizadas por mães diante da internação do filho. A estratégia de solução de problemas mostrou-se a mais presente no relato destas mães, o que significa que apesar de o nascimento de um bebê prematuro e sua consequente internação ser uma situação na qual a mãe tem pouca sensação de controle, ela busca empregar ações instrumentais que contribuam para a alteração deste estressor, seja observando os procedimentos realizados pela equipe, seja buscando participar dos cuidados com o filho. Flores e Trapero (2017) corroboram ao trazer a hipervigilância, a labilidade emocional e até mesmo a impressão de "dissociação" e de pouco impacto afetivo como características que os pais de prematuros podem viver durante a internação. Frisam, inclusive, a importância de não reconhecer essas reações nos pais como patológicas, uma vez que estas auxiliam na construção de um processo de adaptação e de estratégias de enfrentamento frente ao estresse de viver a hospitalização de seu bebê.

Percebe-se, assim, que não apenas impactos psicológicos são vividos pelos pais durante a hospitalização, mas também são possíveis construções de estratégias de enfrentamento em situações de estresse e, consequentemente, um desenvolvimento de resiliência pode ocorrer nesse processo. Rossman, Greene, Kratovil, \& Meier (2017), em um estudo voltado a mães com bebês internados em uma UTIN (Unidade de Terapia Intensiva Neonatal), perceberam que as participantes foram construindo uma importante resiliência a partir do momento em que reconheceram as mudanças em suas vidas, especialmente no puerpério, e aprenderam a conviver com eventos e sentimentos que estavam além de seu controle, dando um novo sentido ao processo de hospitalização e do papel materno. Com isso, os autores acreditam que mães que vivem a experiência de uma UTIN podem viver um crescimento pós-traumático, tendo um novo senso de força, de ressignificação de suas prioridades e de valorização da vida e das experiências como fontes de aprendizados (Rossman et al., 2017).

Consequentemente, pensando na pandemia COVID-19, vamos nos dando conta de algo: os pais de prematuros, talvez por já vivenciarem essa demanda emocional diante da condição de saúde do seu bebê, se mostram, na maioria das vezes, capazes de enfrentar melhor do que nós, equipe de saúde, este momento de pandemia. Já estão mais adaptados com a incerteza, com a fragilidade, já vivem nesse cenário delicado. Por poderem acompanhar os filhos durante a internação, vão adquirindo experiência e compreendendo a dinâmica do hospital e se familiarizando com ela, se adaptando ao processo (Molina et al., 2009). Nesse momento de pandemia, esse processo de familiarização parece contribuir para a percepção de que o hospital possa ser uma opção mais segura de ambiente para o bebê (Fan et al., 2020).

\section{A experiência da atuação na neonatologia durante a pandemia: o fazer da psicologia e os aprendizados com a prematuridade}

Mas e nós, como equipe, como estamos vivendo o momento da pandemia tendo que dar conta dos nossos pacientes, colegas de trabalho e família? Temos medo, inseguranças, fantasias. Mas nós colocamos muito no lugar do outro. Observamos como nos tornamos frágeis também, assim como os bebês e suas famílias. 0 quanto precisamos de uma informação precisa, do acolhimento afetivo e foco para que possamos desenvolver nossas tarefas. Neste momento, nosso tempo parece ser como o vivido pelos pais dos nossos bebês. Não temos uma data de quando tudo vai terminar, de quando iremos retornar às rotinas de trabalho e pessoal, muito parecido com o que escutamos nos relatos das famílias em nossos atendimentos.
Como os pais, será que precisamos de informações precisas ou de acolhimento e afeto? Não seria um exercício vivido pela equipe multiprofissional para que no futuro possam criar outras maneiras de atender e acolher? 0 que desta relação equipe/pais de bebês prematuros e equipe/bebês prematuros ficará após a pandemia de COVID-19? As respostas a essas questões talvez sejam empatia mais pulsante, formas de manejo, cuidados ainda mais colaborativos, pensando no ser humano enquanto sujeito social e não apenas num corpo a ser tratado ou curado (Morsch, Custódio, \& Lamy, 2020).

A rotina da psicologia na Unidade de Neonatologia se transformou, se ampliou. Antes, nosso trabalho centrava-se nas famílias dos bebês, participação de rounds, discussões com equipe multiprofissional. Atualmente, sistematizamos uma rotina de escuta diária da equipe, com o objetivo de identificar demandas de cada turno, acolher eventuais situações mais críticas e mediar conflitos.

Algumas ações específicas foram desenvolvidas, como a comunicação em cada sala das medidas de proteção para os bebês, suas famílias e equipe. Num primeiro momento, percebemos que tanto a equipe como os pais se sentiram seguros, pois todos estavam escutando a mesma informação. E por que a psicologia participava dessa comunicação? A presença do psicólogo fazia com que tanto os pais como profissionais pudessem ser acolhidos no momento da orientação técnica, pois a escuta das medidas protetivas poderia gerar al gum desconforto, principalmente em indivíduos mais frágeis emocionalmente.

Ao longo dos dias, as dúvidas com relação ao uso correto de EPIs começaram a gerar sentimentos diversos nos profissionais. Estudos indicam que além dos impactos psicológicos diretamente ligados ao COVID-19, as medidas de segurança adotadas visando à contenção da pandemia também podem consistir em fatores de risco para a saúde mental dos indivíduos (Schimidit, Crepaldi, Bolze, Neiva-Silva, \& Demenech, 2020). A equipe percebe os equipamentos de segurança como objeto de um movimento de controle, em que, a partir do momento em que os possuem, conseguem administrar 0 ambiente de forma a estar livre de ameaças (Junqueira, 2020). Uma vez que se deparam com a desilusão do controle absoluto, diversas angústias se tornam latentes, e o trabalho da Psicologia vem se fazendo importante para trabalhar essas questões. Além disso, percebe-se, por parte dos profissionais de saúde, que, ainda que estes não estejam atuando diretamente com pacientes diagnosticados com COVID-19, podem apresentar sofrimento psicológico em contextos de emergência de saúde, fenômeno que podemos denominar como traumatização secundária, em que indivíduos que não sofreram diretamente com uma situação, passam a apresentar sintomas decorrentes da empatia por quem sofreu diretamente com a mesma, no caso profissionais atuantes na linha de frente contra o COVID-19 (Brooks et al., 2020; Li et al., 2020). Por um considerável período, enquanto não tínhamos casos suspeitos, percebíamos esses fenômenos circularem pela unidade.

Contudo, surge em um dado momento um novo cenário, já esperado, mas não pouco amedrontador: suspeitas de bebês com CoVID-19. A equipe preocupada em como noticiar para os pais desses bebês e o reflexo disso em toda a unidade. Momento difícil, em que a psicologia desempenhou seu papel auxiliando os demais profissionais na informação e na necessidade dos pais de não comparecerem ao hospital até vir o resultado do exame de seus bebês. Mais uma vez, nos reportamos ao momento do nascimento do bebê prematuro, em que o distanciamento é abrupto e necessário, a necessidade de a mãe aguardar na recuperação para somente após algumas horas rever e reconhecer seu filho. Diante da suspeita de uma infecção viral, reavivam-se 
os sentimentos e impotência, medo de perda, de distanciamento agora por mais horas que já havia experimentado, somados à angústia da espera. Questões que ouvimos desses pais: será que eu passei para o meu bebê? Não tenho nenhum sintoma, como pode ter acontecido isso? Não toco no meu filho para não o colocar em risco. Observo que a equipe se cuida também, como pode acontecer?

Estas perguntas também se estendiam aos profissionais. Nova intervenção psicológica, agora com todos os pais da Unidade e com a equipe que ficaria na sala com os bebês isolados. Um misto de sentimentos foi identificado nesses pais: medo de que seus filhos também pudessem experimentar a mesma situação e a solidariedade com aqueles que precisaram se afastar do hospital.

A equipe que ficaria isolada junto ao plantão também precisava ser acolhida. Não imaginava ter que enfrentar, de maneira surpresa, a tarefa de se paramentarem e ficar até 0 final do plantão diante dessa situação. A escuta a esses profissionais também se deu de forma pontual, focada na entrada e na saída do horário de trabalho. Identificamos que faziam uso de determinadas estratégias, como a atenção redobrada aos cuidados, conversas que não incluíam o momento que estavam passando, algo que fazem diariamente no turno de trabalho e uma organização mental de como iriam até suas residências de forma segura. A escuta dos profissionais da unidade se deu de maneira individual e em grupos das salas em que trabalhavam. A disponibilidade interna da equipe para o enfrentamento, naquele momento, foi observada na rápida organização de como iriam auxiliar os profissionais que não poderia circular na Unidade, naquele momento.

Com a notícia dos exames negativos, a Unidade passou a funcionar novamente com a autorização da presença de todos os pais. Porém, agora, com a liberação apenas de um dos pais com o filho, sem possibilidade do casal estar junto na Unidade, como é a rotina. Novamente a intervenção da psicologia, pois mais uma vez a separação brusca de um dos pais do seu bebê, a separação inesperada do seu filho, como ao nascimento. Tudo que é estranho ao ambiente se busca eliminar, muitas vezes considerando os próprios pais dos bebês como ameaça, já que existe um não saber da equipe em relação à possibilidade de estarem contaminados ou não, buscando, então, privá-los 0 quanto possível de entrarem em seu ambiente supostamente controlado (Junqueira, 2020).

Podemos fazer um paralelo entre as reações dos pais, com os quais intervimos diariamente como rotina, com as reações observadas na equipe. Exaustivamente, os pais são orientados com rotinas, cuidados, como devem manter o comportamento na Unidade junto ao filho. Por vezes, alguns mais angustiados não conseguem assimilar prontamente o que lhes é dito. Repetições, embates, resistências, negação, falta de limites. Atualmente, 0 mesmo comportamento também é percebido em al guns membros da equipe, a resistência para assimilar determinadas orientações (que se modificam conforme a evolução da pandemia) e geram insegurança, conflitos, aumento de ansiedade e impulsividade. Será que a equipe é capaz de reconhecer seu funcionamento tão parecido com o dos pais, que tanto julgamos ou cobramos? Esperamos que, com o passar do tempo, essa reflexão possa ser realizada.

0 olhar para a equipe também se estende para os colaboradores da área administrativa, pensando no hospital como um todo. São as primeiras pessoas com quem os pais têm contato ao entrarem na Unidade, que informam onde está 0 bebê, que solicitam os documentos e, no atual momento de pandemia, se tornam responsáveis por restringir entrada de pessoas que extrapolem as atuais regras de contingenciamento; logo, assumem um papel regulatório na unidade, recebendo a angústia e, em al guns momentos, agressividade dos pais mais abalados pela hospitalização de seus bebês e pelas mudanças em função da pandemia. Esses profissionais também sofrem pelo desconhecido, pelo medo de se contaminarem e pelo cuidado pessoal e com os seus familiares. A escuta psicológica desse grupo também se faz necessária, pois acompanham as orientações, as demandas dos pais e as demandas da equipe. 0 trabalho com esse grupo teve como objetivo propiciar um espaço de escuta e avaliar como estão cuidando da sua saúde mental para vivenciarem o momento de pandemia.

As demandas são inúmeras, e vão se transformando com o passar do tempo e com o curso da pandemia na região. A escuta e o acolhimento se fazem necessários em todos os momentos, com todos os sujeitos que passam pela unidade. A cada novo evento estressor resultante da pandemia, a psicologia se faz presente para buscar construir, junto das famílias e das equipes, as estratégias necessárias para se adaptar, da forma mais saudável possível, aos desafios encontrados. Apesar do desconhecido, vive-se com uma realidade traumática de uma ordem imensurável aos pais dos bebês: lidar com o bebê real e sua necessidade de internação, de acompanhamento médico, as rupturas vividas e a perda do controle constante sobre a evolução de saúde do bebê e os seus cuidados. Percebemos, cada vez mais, a importância de termos um olhar empático sobre estas vivências, que geram sentimentos não muito distintos do que, hoje, em especial, os profissionais de saúde vivem em função da pandemia. Com isso, buscar intervenções que possam construir, junto das equipes, a resiliência que os próprios pais necessitam desenvolver durante a internação de seus bebês. Como Janvier et al. (2016) confirmam, através de suas próprias experiências como profissionais em unidades de neonatologia e pais de crianças que já estiveram internados em UTIN's, muitos aprendizados obtiveram desta experiência, se reinventando para serem pais dos bebês que de fato tiveram, muitas vezes distantes dos bebês ideais, e que transformações obtiveram para sua vida após a internação, ressignificando eventos posteriores de maior estresse e conflitos. Puderam, a partir disso, reconstruir sua história. Tal como nós todos teremos que reconstruir a nossa junto de uma pandemia tão impactante como a do Covid-19.

\section{Considerações finais}

Momentos de pandemia como 0 atual exigem de toda a população, independente do nível de contato com a doença, mudanças bruscas e a convivência com o medo, com incertezas e restrições. Estratégias de enfrentamento se fazem necessárias, apesar de não se ter ainda claro quais são as mais apropriadas e efetivas para lidar com tantos conflitos emocionais e sentimentos diversos e particulares de cada um diante dessa crise.

Os profissionais de saúde não escapam desses desafios, independente da formação, do tempo de experiência e do preparo para lidar com situações como esta. É um novo tipo de adoecimento, uma nova forma de contaminação, que muito ainda é desconhecido, e o não saber intensifica qual quer angústia, ainda mais para quem está na iminência de atuar diretamente com esta doença. É um processo de se descobrir e aprender a cada dia, a cada informação nova, dentro de um tempo que não se tem definido. Talvez se faça importante ser um pouco "pais" de bebês prematuros, que vivem algo semelhante ao que todos estão vivendo a partir chegada de seu filho antes do tempo.

Tais questões não anulam a importância de um suporte psicossocial, especialmente neste momento de pandemia. A saúde física e mental dos 
profissionais de saúde é de fundamental atenção, especialmente em um contexto de pandemia, e para tanto, 0 apoio psicológico e social neste momento se faz muito necessário (Wang, Qi, Bao, Li, \& Shi, 2020). Tal como em diversas unidades de neonatologia o psicólogo tem importante atuação junto aos pais e seus bebês, os profissionais de saúde necessitam de foco na atenção psicossocial, uma vez que o desafio emocional desta pandemia é grande (Morsh et al., 2020) e pode exceder, muitas vezes, a capacidade estrutural da equipe de se adaptar de forma independente, sem suporte.

É importante frisar que este trabalho é um relato de experiência, e que não é passível de generalizações. As observações realizadas não garantem uma certeza sobre a unanimidade dos pais quanto às suas resiliências e estratégias de enfrentamento. Como Carvalho e Pereira (2017) abordam em seu estudo, a forma como os pais utilizam de estratégias de enfrentamento varia conforme os aspectos psicodinâmicos de cada um, o que envolve a história de vida individual, a rede de apoio, entre outros.

Buscamos, aqui, instigar reflexões, aguçar a atenção a determinadas situações que possam ter algo a contribuir com as intervenções de saúde mental neste momento, a fim de torná-las mais assertivas e efetivas. Esperamos que novas pesquisas possam surgir dessas reflexões. Assim, pensando que os bebês prematuros já vivem o distanciamento social, fica ainda o questionamento: 0 que eles têm a nos ensinar neste momento?

\section{Referências}

Brooks, S. K., Webster, R. K., Smith, L. E., Woodland, L., Wessely, S., Greenberg, N., \& Rubin, G. J. (2020). The psychological impact of quarantine and how to reduce it: rapid review92020 of the evidence. The Lancet, 395(10227), 14-20. doi: 10.1016/50140-6736(20)30460-8.

Carvalho, L. D. S., \& Pereira, C. D. M. C. (2017). As reações psicológicas dos pais frente à hospitalização do bebê prematuro na UTI neonatal. Revista da SBPH, 20(2), 101-122. Recuperado em 03 de maio, 2020, de http://pepsic.bvsalud.org/scielo.php?script=sci_arttext\&pi$d=S 1516-08582017000200007$

Fan, J., Zhou, M., Wei, L., Fu, L., Zhang, X., \& Shi, Y. A Qualitative Study on the Psychological Needs of Hospitalized Newborns'Parents During COVID-19 Outbreak in China. Iranian Journal of Pediatrics, 30(2). doi: 10.5812/ ijp. 102748.

Flores, I. C., \& Trapero, M. V. (2017). Programa de Atención Psicológica en Neonatología: Experiencia del Hospital Clínico San Carlos de Madrid Psychological Care Program in Neonatology: The Hospital Clínico San Carlos's experience. Revista Clínica Contemporánea, 8(28), 1-12. doi: 10.5093/cc2017a18

Gil, A.C. (2002). Como elaborar projetos de pesquisa. São Paulo: Atlas.

Janvier, A., Lantos, J., Aschner, J., Barrington, K., Batton, B., Batton, D., ... \& Lyerly, A. D. (2016). Stronger and more vulnerable: a balanced view of the impacts of the NICU experience on parents. Pediatrics, 138(3). doi: 10.1542/peds.2016-0655

John Hopkins University and Medicine (2020, julho 09). COVID-19 Dashboard. Baltimore: John Hopkins University and Medicine. Recuperado de https:// coronavirus.jhu.edu/map.html

Junqueira, F. (2020, abril 23). Encontro COVID-19: Orientações para maternidades e unidades neonatais. Rio de Janeiro: IFF/Fiocruz. Recuperado de https://portaldeboaspraticas.iff.fiocruz.br/especialista/COVID-19-orientacoes-para-maternidades-e-unidades-neonatais/.
Li, W., Yang, Y., Liu, Z. H., Zhao, Y. J., Zhang, Q., Zhang, L., Cheung, T., \& Xiang, Y. T. (2020). Progression of mental health services during the COVID-19 outbreak in China. International Journal of Biological Sciences, 16(10), 1732. doi: $10.7150 /$ ijbs. 45120.

Loss, A. B. M., Caprini, F. R., Rigoni, P. V. M. D. S., \& Andrade, B. L. S. D. (2015). Estados emocionais e estratégias de enfrentamento de mães de recém-nascidos de risco. Gerais: Revista Interinstitucional de Psicologia, 8(1),03-18. Recuperado em 01 de maio, 2020, de http://pepsic.bvsalud. org/scielo.php?script=sci_arttext\&pid=\$1983-82202015000100002.

Ministério da Saúde (2020, jul 09). Painel Coronavírus. Brasília: Ministério da Saúde. Recuperado de https://covid.saude.gov.br/

Molina, R. C. M., Fonseca, E. L., Waidman, M. A. P., \& Marcon, S. S. (2009). A percepção da família sobre sua presença em uma Unidade de Terapia Intensiva Pediátrica e Neonatal. Revista da Escola de Enfermagem da USP, 43(3), 630-638. doi: 10.1590/50080-62342009000300019.

Morsch, D. S., Custódio, Z. A. D. 0., \& Lamy, Z. C. (2020). Cuidados psicoafetivos em unidade neonatal diante da pandemia de COVID-19. Revista Paulista de Pediatria, 38. Recuperado de https://www.scielo.br/scielo. php?pid=S0103-05822020000100102\&script=sci_arttext\&tlng=pt

Organização Pan-Americana de Saúde, \& Organização Mundial da Saúde (2020). Folhalnformativa - COVID-19 (Doença Causada Pelo Novo Coronavírus). Recuperado de https://www.paho.org/bra/index.php?option=com_content\&view $=$ article\&id $=6101:$ covid19\&ltemid $=875$

Rosa, R. R., \& Gill, M. E. (2017). Suporte psicológico aos pais na Unidade de Tratamento Intensivo Neonatal: encontros possíveis e necessários. Revista daSBPH, 20(2), 123-135. Recuperado de http://pepsic.bvsalud.org/scielo. php?script=sci_arttext\&pid=\$1516-08582017000200008

Rossman, B., Greene, M. M., Kratovil, A. L., \& Meier, P. P. (2017). Resilience in mothers of very-low-birth-weight infants hospitalized in the NICU. Journal of Obstetric, Gynecologic \& Neonatal Nursing, 46(3), 434-445. doi: 10.1016/j.jogn.2016.11.016

Schmidt, B., Crepaldi, M. A., Bolze, S. D. A., Neiva-Silva, L., \& Demenech, L. M. (2020). Impactos na Saúde Mental e Intervenções Psicológicas Diante da Pandemia do Novo Coronavírus (COVID-19). Scielo Preprints, 2020. doi: 10.1590/SciELOPreprints.58.

Silva, R. M. M., da Silva Menezes, C. C., Cardoso, L. L., \& França, A. F. O. (2016). Vivências de famílias de neonatos prematuros hospitalizados em unidade de terapia intensiva neonatal: revisão integrativa. Revista de Enfermagem do Centro-Oeste Mineiro, 6(2), 2258-2270. doi: 10.19175/recom.v6i2.940.

Wang, J., Qi, H., Bao, L., Li, F., \& Shi, Y. (2020). A contingency plan for the management of the 2019 novel coronavirus outbreak in neonatal intensive care units. The Lancet Child \& Adolescent Health, 4(4), 258-259. doi: 10.1016/S2352-4642(20)30040-7 